\title{
An Explicit upper Bound of the Argument of Dirichlet $L$-functions on the Generalized Riemann Hypothesis
}

\author{
Takahiro Wakasa* \\ Hachinohe National College of Technology, Uwanotai, Tamonoki, Hachinoheshi, Aomoriken, Japan \\ *Corresponding author: wakasa-g@hachinohe-ct.ac.jp
}

Received August 13, 2015; Revised September 30, 2015; Accepted October 08, 2015

\begin{abstract}
We prove an explicit upper bound of the function $S(t, \chi)$, defined by the argument of Dirichlet $L$-functions attached to a primitive Dirichlet character $\chi(\bmod q>1)$. An explicit upper bound of the function $S(t)$, defined by the argument of the Riemann zeta-function, have been obtained by A. Fujii [1]. Our result is obtained by applying the idea of Fujii's result on $S(t)$. The constant part of the explicit upper bound of $S(t, \chi)$ in this paper does not depend on $\chi$. Our proof does not cover the case $q=1$ and indeed gives a better bound than the one of Fujii that covers the case $q=1$.
\end{abstract}

Keywords: Dirichlet L-functions

Cite This Article: Takahiro Wakasa, "An Explicit upper Bound of the Argument of Dirichlet $L$-functions on the Generalized Riemann Hypothesis." Turkish Journal of Analysis and Number Theory, vol. 3, no. 5 (2015): 140-144. doi: 10.12691/tjant-3-5-5.

\section{Introduction}

We consider the argument of Dirichlet $L$-functions. Let $L(s, \chi)$ be the Dirichlet $L$-function, where $s=\sigma+i t$ is a complex variable, associated with a primitive Dirichlet character $\chi(\bmod q>1)$. We denote the non-trivial zeros of $L(s, \chi)$ by $\rho(\chi)=\beta(\chi)+i \gamma(\chi)$ where $\beta(\chi)$ and $\gamma(\chi)$ are real numbers. Then, when $t$ is not the ordinate of a zero of $L(s, \chi)$, we define

$$
S(t, \chi)=\frac{1}{\pi} \arg L\left(\frac{1}{2}+i t, \chi\right) .
$$

This is given by continuous variation along the straight line $s=\sigma+i t$, as $\sigma$ varies from $+\infty$ to $\frac{1}{2}$, starting with the value zero. Also, when $t$ is the ordinate of a zero of $L(s, \chi)$, we define

$$
S(t, \chi)=\frac{1}{2}\{S(t+0, \chi)+S(t-0, \chi)\} .
$$

In Selberg [2], it is known that

$$
S(t, \chi)=O(\log q(t+1))
$$

and under the generalized Riemann hypothesis (GRH)

$$
S(t, \chi)=O\left(\frac{\log q(t+1)}{\log \log q(t+3)}\right) .
$$

Concerning the constant in $O$-notation, the indication that, for $t \rightarrow \infty$, a formula is valid uniformly in $q>q_{0}$.

The purpose of the present article is to prove the following result.
Theorem 1. Assuming GRH. Then, for $q>1$

$$
|S(t, \chi)|<0.804 \cdot \frac{\log q(t+1)}{\log \log q(t+3)}+O\left(\frac{\log q(t+3)}{\log \log q(t+3)^{2}}\right) \text {. }
$$

The constant 0:804 obviously does not depend on $\chi$. And we don't know anything concerning the optimality. Also, the implied constant of the error term does not depend on $q$. The details of the argument concerning error terms can be seen in the proof of this theorem. However, our result does not include the case of the function $S(t)$ which is defined by the argument of the Riemann zetafunction since we assume $q>1$. An explicit upper bound of the function $S(t)$ is obtained by A. Fujii [1], where the value is 0.83 .

The basic policy of the proof of this theorem is based on A. Fujii [1]. In the proof, $S(t, \chi)$ is seperated by three parts $M_{1}, M_{2}$ and $M_{3}$. Fujii's idea of [1] is applied to all parts. But we need Lemma 1, which is an explicit formula for $\frac{L^{\prime}}{L}(s, \chi)$. This lemma is an analogue of Selberg's result.

But the constant part of Theorem 1 is not best result for $S(t, \chi)$. In 2007, D. A. Goldston, S. M. Gonek [7] proved following result;

$$
|S(t)|<\left(\frac{1}{2}+o(1)\right) \frac{\log t}{\log \log t}
$$

for $t \rightarrow \infty$ under the Riemann Hypothesis. This result suggest that analogous bound could be true also for the Dirichlet $L$-function.

Also, E. Carneiro, V. Chandee, M. B. Milinovich [5] proved

$$
|S(t)|<\frac{1}{4} \cdot \frac{\log t}{\log \log t}+O\left(\frac{\log t \log \log \log t}{(\log \log t)^{2}}\right)
$$


and

$$
-\left(\frac{\pi}{24}+o(1)\right) \frac{\log t}{(\log \log t)^{2}} \leq S_{1}(t) \leq\left(\frac{\pi}{48}+o(1)\right) \frac{\log t}{(\log \log t)^{2}}
$$

in 2013.

Thus, for the Riemann zeta-function, Fujii's result was improved by D. A. Goldston, S. M. Gonek [7], after that the result was improved by E. Carneiro, V. Chandee, M. B. Milinovich [5]. Moreover, for a vast class of general $L$ function (in particular, for $L(s, \chi)$ ), those better constant are $\frac{1}{4}$ established by E. Carneiro, V. Chandee, M. B. Milinovich [6] under GRH.

So, the main result of this paper is a particular case of the work [6]. Therefore, Theorem 1 is given by an alternative proof in the case of Dirichlet $L$-function with a weaker constant using a different method.

In future, we will approach to obtain an explicit upper bound of the multiple integral of the Dirichlet $L$-function under the GRH based on ideas of [5].

\section{Some Notations and a Lemma}

To prove our result, we introduce some notations and prove the aforementioned Lemma 1.

Let $s=\sigma+i t$. We suppose that $\sigma \geq \frac{1}{2}$ and $t \geq 2$. Let $x$ be a positive number satisfying $4 \leq x \leq t^{2}$. Also, we put

$$
\sigma_{1}=\frac{1}{2}+\frac{1}{\log x}
$$

and

with

$$
\Lambda_{x}(n)= \begin{cases}\Lambda(n) & \text { for } 1 \leq n \leq x, \\ \Lambda(n) \frac{\log \frac{x^{2}}{n}}{\log x} & \text { for } x \leq n \leq x^{2},\end{cases}
$$

$\Lambda(n)= \begin{cases}\log p & \text { if } n=p^{k} \text { with a prime } p \text { and an integer } k \geq 1, \\ 0 & \text { otherwise. }\end{cases}$

Using these notations, we prove the following lemma.

Lemma 1. Assume the GRH. Let $t \geq 2$ and $x>0$ such that $4 \leq x \leq t^{2}$. Then for $\sigma \geq \sigma_{1}=\frac{1}{2}+\frac{1}{\log x}$, there exist $\omega$ and $\omega^{\prime}$ such that $|\omega| \leq 1$ and $-1 \leq \omega^{\prime} \leq 1$, we have

$$
\begin{aligned}
& \frac{L^{\prime}}{L}(\sigma+i t, \chi) \\
& =-\sum_{n<x^{2}} \frac{\Lambda_{x}(n)}{n^{\sigma+i t}} \chi(n)-\frac{x^{\frac{1}{2}-\sigma}\left(1+x^{\frac{1}{2}}\right) \omega}{1-\frac{1}{e}\left(1+\frac{1}{e}\right) \omega^{\prime}} \Re\left(\sum_{n<x^{2}} \frac{\Lambda_{x}(n)}{n^{\sigma_{1}+i t}} \chi(n)\right) \\
& +\frac{x^{\frac{1}{2}-\sigma}\left(1+x^{\frac{1}{2}-\sigma}\right) \omega}{1-\frac{1}{e}\left(1+\frac{1}{e}\right) \omega^{\prime}} \cdot \frac{1}{2} \log q(t+1)+O\left(x^{\frac{1}{2}-\sigma}\right) .
\end{aligned}
$$

This is an analogue of Lemma 2 of A. Fujii [1].

Lemma 2. Let $a=0$ if $\chi(-1)=1$, and $a=1$ if $\chi(-1)=-1$. Then, for $x>1, \quad s \neq-2 q-a \quad(q=0,1,2, \ldots)$ and $s \neq \rho(\chi)$, we have

$$
\begin{aligned}
& \frac{L^{\prime}}{L}(s, \chi)=-\sum_{n<x^{2}} \frac{\Lambda_{x}(n)}{n^{s}} \chi(n) \\
& +\frac{1}{\log x} \sum_{q=0}^{\infty} \frac{x^{-2 q-a-s}-x^{-2(2 q+a+s)}}{(2 q+a+s)^{2}} \\
& +\frac{1}{\log x} \sum_{\rho} \frac{x^{\rho-s}-x^{2(\rho-s)}}{(s-\rho)^{2}}
\end{aligned}
$$

Lemma 2 is similar to Lemma 15 of Selberg [2]. We write here only a sketch of the proof of Lemma 2 .

If $a=\max (1, \sigma)$, we have

$$
\begin{aligned}
& \sum_{n<x^{2}} \frac{\Lambda_{x}(n)}{n^{s}} \chi(n) \\
& =\frac{1}{2 \pi i \log x} \int_{a-\infty i}^{a+\infty i} \frac{x^{z-s}-x^{2(z-s)}}{(z-s)^{2}} \cdot \frac{L^{\prime}}{L}(z, \chi) d z .
\end{aligned}
$$

We consider residues which we encounter when we move the path of integration to the left. At the point $z=S$, the residue is $-(\log x) \frac{L^{\prime}}{L}(s, \chi)$. At the zeros $-2 q-a$ $(q=0,1,2, \ldots)$, the residues are $\frac{x^{-2 q-a-s}-x^{-2(2 q+a+s)}}{(2 q+a+s)^{2}}$. At the zeros $s=\rho$ of $L(s, \chi)$, the residues are $\frac{x^{\rho-s}-x^{2(\rho-s)}}{(s-\rho)^{2}}$. Thus, we obtain Lemma 2.

Proof of Lemma 1. Assume the GRH. In Lemma 2, since for $\sigma \geq \sigma_{1}=\frac{1}{2}+\frac{1}{\log x}$

$$
\begin{aligned}
& \left|\frac{1}{\log x} \sum_{\rho} \frac{x^{\rho-s}-x^{2(\rho-s)}}{(s-\rho)^{2}}\right| \\
& =\frac{1}{\log x}\left|\sum_{\gamma} \frac{\left.x^{\left(\frac{1}{2}-\sigma\right)} \mid x^{(\gamma-t) i}-x^{\left(\frac{1}{2}-\sigma\right)+(\gamma-t) i}\right)}{\left(\sigma-\frac{1}{2}\right)^{2}+(t-\gamma)^{2}}\right| \\
& \leq \frac{x^{\left(\frac{1}{2}-\sigma\right)}}{\log x} \sum_{\gamma} \frac{1+x^{\left(\frac{1}{2}-\sigma\right)}}{\left(\sigma-\frac{1}{2}\right)^{2}+(t-\gamma)^{2}} \mid \\
& \leq x^{\left(\frac{1}{2}-\sigma\right)} \mid \frac{\left(\frac{1}{2}-\sigma\right)}{1+x^{2}} \sum_{\gamma} \frac{\sigma_{1}-\frac{1}{2}}{\left(\sigma_{1}-\frac{1}{2}\right)^{2}+(t-\gamma)^{2}}
\end{aligned}
$$


we have

$$
\begin{aligned}
& \frac{1}{\log x} \sum_{\rho} \frac{x^{\rho-s}-x^{2(\rho-s)}}{(s-\rho)^{2}} \\
& =x^{\frac{1}{2}-\sigma}\left(1+x^{\frac{1}{2}-\sigma}\right) \omega \sum_{\gamma} \frac{\sigma_{1}-\frac{1}{2}}{\left(\sigma_{1}-\frac{1}{2}\right)^{2}+(t-\gamma)^{2}},
\end{aligned}
$$

where $|\omega|<1$. Hence Lemma 2 can be rewritten by

$$
\begin{aligned}
& \frac{L^{\prime}}{L}(\sigma+i t, \chi)=\sum_{n<x^{2}} \frac{\Lambda_{x}(n)}{n^{\sigma+i t}} \chi(n)+O\left(\frac{x^{\frac{1}{2}-\sigma}}{\log x}\right) \\
& +x^{\frac{1}{2}-\sigma}\left(1+x^{\frac{1}{2}-\sigma}\right) \omega \sum_{\gamma} \frac{\sigma_{1}-\frac{1}{2}}{\left(\sigma_{1}-\frac{1}{2}\right)^{2}+(t-\gamma)^{2}}
\end{aligned}
$$

for $\sigma \geq \sigma_{1}$.

In particular, since $x^{\frac{1}{2}-\sigma} \leq x^{-\frac{1}{\log x}}=\frac{1}{e}$ for $\sigma \geq \sigma_{1}$, we get

$$
\begin{aligned}
\Re \frac{L^{\prime}}{L}\left(\sigma_{1}+i t, \chi\right) & =-\Re\left(\sum_{n<x^{2}} \frac{\Lambda_{x}(n)}{n^{\sigma_{1}+i t}} \chi(n)\right)+O\left(\frac{1}{\log x}\right) \\
& +\frac{1}{e}\left(1+\frac{1}{e}\right) \omega^{\prime} \sum_{\gamma} \frac{\sigma_{1}-\frac{1}{2}}{\left(\sigma_{1}-\frac{1}{2}\right)^{2}+(t-\gamma)^{2}},
\end{aligned}
$$

where $-1 \leq \omega^{\prime} \leq 1$.

Here, since by p. 46 of Selberg [2]

$$
\begin{aligned}
\Re \frac{L^{\prime}}{L}(s, \chi) & =\Re\left(-\frac{1}{2} \log \frac{q}{\pi}-\frac{1}{2} \log \left(\frac{s+a}{2}\right)\right) \\
& +\sum_{\gamma} \frac{\sigma-\frac{1}{2}}{\left(\sigma-\frac{1}{2}\right)^{2}+(t-\gamma)^{2}}+O(1),
\end{aligned}
$$

we get for $t \geq 2$

$$
\begin{aligned}
& \Re \frac{L^{\prime}}{L}\left(\sigma_{1}+i t, \chi\right) \\
& =-\frac{1}{2} \log q(t+1)+\sum_{\gamma} \frac{\sigma_{1}-\frac{1}{2}}{\left(\sigma_{1}-\frac{1}{2}\right)^{2}+(t-\gamma)^{2}}+O(1) .
\end{aligned}
$$

By (2) and (3) we have

$$
\begin{aligned}
& \left(1-\frac{1}{e}\left(1+\frac{1}{e}\right) \omega^{\prime}\right) \sum_{\gamma} \frac{\sigma_{1}-\frac{1}{2}}{\left(\sigma_{1}-\frac{1}{2}\right)^{2}+(t-\gamma)^{2}} \\
& =-\Re\left(\sum_{n<x} \frac{\Lambda_{x}(n)}{n^{\sigma_{1}+i t}} \chi(n)\right)+\frac{1}{2} \log q(t+1)+O\left(\frac{1}{\log x}\right)+O(1) .
\end{aligned}
$$

Inserting the above inequality to (1), we obtain Lemma 1.

\section{Proof of Theorem 1}

The quantity $S(t, \chi)$ is separated into the following three parts.

$$
\begin{aligned}
& S(t, \chi)=-\frac{1}{\pi} \int_{\frac{1}{2}}^{\infty} \mathfrak{I} \frac{L^{\prime}}{L}(\sigma+i t, \chi) d \sigma \\
& =-\frac{1}{\pi}\left\{\begin{array}{l}
\mathfrak{I} \int_{\sigma_{1}}^{\infty} \frac{L^{\prime}}{L}(\sigma+i t, \chi) d \sigma \\
+\mathfrak{I}\left\{\left(\sigma_{1}-\frac{1}{2}\right) \frac{L^{\prime}}{L}\left(\sigma_{1}+i t, \chi\right)\right\} \\
-\mathfrak{I} \int_{\sigma_{1}}^{\infty}\left\{\frac{L^{\prime}}{L}\left(\sigma_{1}+i t, \chi\right)-\frac{L^{\prime}}{L}(\sigma+i t, \chi)\right\} d \sigma
\end{array}\right\} \\
& =-\frac{1}{\pi} \mathfrak{I}\left(M_{1}+M_{2}+M_{3}\right),
\end{aligned}
$$

say.

First, we estimate $M_{1}$. By Lemma 1 we have

$$
\begin{aligned}
& M_{1} \\
& =\int_{\sigma_{1}}^{\infty}\left\{\begin{array}{c}
-\sum_{n<x^{2}} \frac{\Lambda_{x}(n)}{n^{\sigma+i t}} \chi(n)-\frac{x^{\frac{1}{2}-\sigma}\left(1+x^{\frac{1}{2}-\sigma}\right)}{1-\frac{1}{e}\left(1+\frac{1}{e}\right) \omega^{\prime}} \\
\times\left(\sum_{n<x^{2}} \frac{\Lambda_{x}(n)}{n^{\sigma_{1}+i t}} \chi(n)\right) \\
+\frac{x^{\frac{1}{2}-\sigma}\left(1+x^{\frac{1}{2}}\right) \omega}{1-\frac{1}{e}\left(1+\frac{1}{e}\right) \omega^{\prime}} \cdot \frac{1}{2} \log q(t+1)+O\left(x^{\frac{1}{2}-\sigma}\right)
\end{array}\right\} d \sigma \\
& =-\int_{\sigma_{1}}^{\infty} \sum_{n<x^{2}} \frac{\Lambda_{x}(n)}{n^{\sigma+i t}} \chi(n) d \sigma+\eta_{1}(t) \\
& =-\sum_{n<x^{2}} \frac{\Lambda_{x}(n)}{n^{\sigma_{1}+i t} \log n} \chi(n)+\eta_{1}(t) \text {, }
\end{aligned}
$$

say. Here,

$$
\begin{aligned}
&\left|\eta_{1}(t)\right|=\left|\int_{\sigma_{1}}^{\infty} \frac{\Re_{\omega}\left(1+x^{\frac{1}{2}-\sigma}\right) x^{\frac{1}{2}-\sigma}}{1-\frac{1}{e}\left(1+\frac{1}{e}\right) \omega^{\prime}} d \sigma\right| \\
& \cdot\left|\mathfrak{R}\left(\sum_{n<x^{2}} \frac{\Lambda_{x}(n)}{n^{\sigma}+i t} \chi(n)\right)-\frac{1}{2} \log q(t+1)\right| \\
&+O\left(\int_{\sigma_{1}}^{\infty} x^{\frac{1}{2}-\sigma} d \sigma\right)
\end{aligned}
$$




$$
\begin{aligned}
& \leq \frac{1}{1-\frac{1}{e}\left(1+\frac{1}{e}\right)}\left|\Re\left(\sum_{n<x^{2}} \frac{\Lambda_{x}(n)}{n^{\sigma_{1}+i t}} \chi(n)\right)-\frac{1}{2} \log q(t+1)\right| \\
& \quad \times \int_{\sigma_{1}}^{\infty} x^{\frac{1}{2}-\sigma}\left(1+x^{\frac{1}{2}-\sigma}\right) d \sigma+O\left(\int_{\sigma_{1}}^{\infty} x^{\frac{1}{2}-\sigma} d \sigma\right) \\
& \leq \frac{\left(\frac{1}{e}+\frac{1}{2 e^{2}}\right)}{1-\frac{1}{e}\left(1+\frac{1}{e}\right)} \cdot \frac{1}{2} \cdot \frac{\log q(t+1)}{\log x} \\
& +O\left(\frac{1}{\log x}\left|\sum_{n<x^{2}} \frac{\Lambda_{x}(n)}{n^{\sigma_{1}+i t}} \chi(n)\right|\right),
\end{aligned}
$$

say.

Next, applying Lemma 1 to $M_{2}$, we get

$$
\left.M_{2}=\mid \begin{array}{l}
-\sum_{n<x^{2}} \frac{\Lambda_{x}(n)}{n^{\sigma_{1}+i t}} \chi(n) \\
-\frac{x^{\frac{1}{2}-\sigma_{1}}\left(1+x^{\frac{1}{2}-\sigma_{1}}\right) \omega}{1-\frac{1}{e}\left(1+\frac{1}{e}\right) \omega^{\prime}} \\
\log x \\
+\frac{x^{\frac{1}{2}-\sigma_{1}}\left(1+x^{\frac{1}{2}-\sigma_{1}}\right) \omega}{1-\frac{1}{e}\left(1+\frac{1}{e}\right) \omega^{\prime}} \log q(t+1) \\
\left.x^{\frac{1}{2}-\sigma_{1}}\right)
\end{array}\right\}
$$$$
\begin{aligned}
& \leq \frac{\left(\frac{1}{e}+\frac{1}{2 e^{2}}\right)}{1-\frac{1}{e}\left(1+\frac{1}{e}\right)} \cdot \frac{1}{2} \cdot \frac{\log q(t+1)}{\log x} \\
& +O\left(\frac{1}{\log x}\left|\sum_{n<x^{2}} \frac{\Lambda_{x}(n)}{n^{\sigma_{1}+i t}} \chi(n)\right|\right),
\end{aligned}
$$

say.

Next we estimate $M_{3}$. By Lemma 16 of Selberg [2] we get

$$
\begin{aligned}
& \left|\mathfrak{J}\left(M_{3}\right)\right|=\left|\int_{\frac{1}{2}}^{\sigma_{1}} \mathfrak{J}\left\{\begin{array}{l}
\sum_{\rho} \frac{1}{\sigma_{1}+i t-\rho} \\
-\sum_{\rho} \frac{1}{\sigma+i t-\rho}+O(1)
\end{array}\right\} d \sigma\right| \\
& \leq\left|\int_{\frac{1}{2}}^{\sigma_{1}} \sum_{\gamma} \frac{(t-\gamma)\left\{\left(\sigma-\frac{1}{2}\right)^{2}-\left(\sigma_{1}-\frac{1}{2}\right)^{2}\right\}}{\left\{\left(\sigma_{1}-\frac{1}{2}\right)^{2}+(t-\gamma)^{2}\right\}\left\{\left(\sigma-\frac{1}{2}\right)^{2}+(t-\gamma)^{2}\right\}} d \sigma\right| \\
& +O \frac{1}{\log x} \\
& <\int_{\frac{1}{2}}^{\sigma_{1}} \sum_{\gamma} N(\gamma, \sigma) d \sigma+O \frac{1}{\log x},
\end{aligned}
$$

say.

Here, we put $\Re=\int_{\frac{1}{2}}^{\sigma_{1}} \sum_{\gamma} N(\gamma, \sigma) d \sigma$. If $t=\gamma$, we see $\Re=0$ easily. If $t \neq \gamma$, we have

$$
\begin{aligned}
& \Re<\sum_{\gamma} \frac{\left(\sigma_{1}-\frac{1}{2}\right)^{2}}{\left(\sigma_{1}-\frac{1}{2}\right)^{2}+(t-\gamma)^{2}} \int_{\frac{1}{2}}^{\sigma_{1}} \frac{|t-\gamma|}{\left(\sigma-\frac{1}{2}\right)^{2}+(t-\gamma)^{2}} d \sigma \\
& \leq \sum_{\gamma} \frac{\left(\sigma_{1}-\frac{1}{2}\right)^{2}}{\left(\sigma_{1}-\frac{1}{2}\right)^{2}+(t-\gamma)^{2}} \int_{\frac{1}{2}}^{\infty} \frac{|t-\gamma|}{\left(\sigma-\frac{1}{2}\right)^{2}+(t-\gamma)^{2}} d \sigma
\end{aligned}
$$$$
\leq \frac{\pi}{2 \log x} \sum_{\gamma} \frac{\left(\sigma_{1}-\frac{1}{2}\right)^{2}}{\left(\sigma_{1}-\frac{1}{2}\right)^{2}+(t-\gamma)^{2}}
$$

since $\sigma<\sigma_{1}$ for $M_{3}$.

Here, by (2) and (3) we get

$$
\begin{aligned}
& \sum_{\gamma} \frac{\left(\sigma_{1}-\frac{1}{2}\right)^{2}}{\left(\sigma_{1}-\frac{1}{2}\right)^{2}+(t-\gamma)^{2}} \\
& =\frac{1}{1-\frac{1}{e}\left(1+\frac{1}{e}\right) \omega^{\prime}} \cdot \frac{1}{2} \log q(t+1) \\
& +O\left(\left|\sum_{n<x^{2}} \frac{\Lambda_{x}(n)}{n^{\sigma_{1}+i t}} \chi(n)\right|\right)+O\left(\frac{1}{(\log x)^{2}}\right) .
\end{aligned}
$$

So,

$$
\begin{aligned}
& \Re=\frac{1}{4} \cdot \frac{1}{1-\frac{1}{e}\left(1+\frac{1}{e}\right) \omega^{\prime}} \cdot \frac{1}{\log x} \cdot \log q(t+1) \\
& +O\left(\frac{1}{\log x}\left|\sum_{n<x^{2}} \frac{\Lambda_{x}(n)}{n^{\sigma_{1}+i t}} \chi(n)\right|\right)+O\left(\frac{1}{(\log x)^{3}}\right) .
\end{aligned}
$$

Hence we have

$$
\begin{aligned}
& \left|\mathfrak{I}\left(M_{3}\right)\right| \leq \frac{1}{4} \cdot \frac{1}{1-\frac{1}{e}\left(1+\frac{1}{e}\right) \omega^{\prime}} \cdot \frac{1}{\log x} \cdot \log q(t+1) \\
& \quad+O\left(\frac{1}{\log x}\left|\sum_{n<x^{2}} \frac{\Lambda_{x}(n)}{n^{\sigma_{1}+i t}} \chi(n)\right|\right)+O\left(\frac{1}{\log x}\right) \\
& =\eta_{4}(t)+O\left(\frac{1}{\log x}\left|\sum_{n<x^{2}} \frac{\Lambda_{x}(n)}{n^{\sigma_{1}+i t}} \chi(n)\right|\right)+O\left(\frac{1}{\log x}\right) .
\end{aligned}
$$

say.

Finally, we estimate the sums on right-hand sides of (4), (5), (6) and (7). By definition of $\Lambda_{X}(n)$ we have 


$$
\begin{aligned}
& \left|\sum_{n<x^{2}} \frac{\Lambda_{x}(n)}{n^{\sigma_{1}+i t}} \chi(n)\right| \\
& \leq \sum_{n<x} \frac{\Lambda_{x}(n)}{n^{\frac{1}{2}}}+\sum_{x \leq n \leq x^{2}} \frac{\Lambda_{x}(n) \log \frac{x^{2}}{n}}{n^{\frac{1}{2}}} \cdot \frac{1}{\log x} \ll \frac{x}{\log x} .
\end{aligned}
$$

Similarly,

$$
\left|\sum_{n<x^{2}} \frac{\Lambda_{x}(n)}{n^{\sigma_{1}+i t} \log n} \chi(n)\right| \ll \frac{x}{(\log x)^{2}} .
$$

So, we see

$$
\begin{aligned}
& \left|M_{1}\right| \leq \frac{\left(\frac{1}{e}+\frac{1}{2 e^{2}}\right)}{1-\frac{1}{e}\left(1+\frac{1}{e}\right)} \cdot \frac{1}{2} \cdot \frac{\log q(t+1)}{\log x}+O\left(\frac{x}{(\log x)^{2}}\right), \\
& \left|M_{2}\right| \leq \frac{\left(\frac{1}{e}+\frac{1}{2 e^{2}}\right)}{1-\frac{1}{e}\left(1+\frac{1}{e}\right)} \cdot \frac{1}{2} \cdot \frac{\log q(t+1)}{\log x}+O\left(\frac{x}{(\log x)^{2}}\right),
\end{aligned}
$$

and

$$
\left|M_{3}\right| \leq \eta_{4}(t)+O\left(\frac{x}{(\log x)^{2}}\right)
$$

For $\eta_{1}(t), \quad \eta_{2}(t), \quad \eta_{3}(t)$ and $\eta_{4}(t)$, taking $x=\log q(t+3) \sqrt{\log q(t+3)}$ we have

$$
\begin{aligned}
& |S(t, \chi)|<\frac{1}{\pi} \cdot \frac{1}{1-\frac{1}{e}\left(1+\frac{1}{e}\right)}\left\{\begin{array}{l}
\frac{\left(\frac{1}{e}+\frac{1}{2 e^{2}}\right)}{2} \\
+\frac{\left(\frac{1}{e}+\frac{1}{e^{2}}\right)}{2}+\frac{\pi}{4}
\end{array}\right\} \frac{\log q(t+1)}{\log x} \\
& +O\left(\frac{x}{(\log x)^{2}}\right) \\
& =0.803986 \cdots \frac{\log q(t+1)}{\log \log q(t+3)}+O\left(\frac{\log q(t+3)}{(\log \log q(t+3))^{2}}\right) .
\end{aligned}
$$

Therefore we obtain the theorem.

\section{Acknowledgments}

I thank Prof. Kohji Matsumoto for his advice and patience during the preparation of this paper. I also thank Prof. Giuseppe Molteni, Prof. Yumiko Umegaki and Dr. Ryo Tanaka, who gave many important advice. Finally, I thank the referee who indicates errors in this paper.

\section{References}

[1] A. Fujii, An explicit estimate in the theory of the distribution of the zeros of the Riemann zeta function, Comment. Math. Univ. Sancti Pauli, 53, (2004), 85-114.

[2] A. Selberg, Contributions to the theory of Dirichlet's L-function, Avh. Norske Vir. Akad. Oslo I:1, (1946), No. 3, 1-62.

[3] A. Selberg, Collected Works, vol I, 1989, Springer.

[4] E. C. Titchmarsh, The theory of the Riemann zeta-function, Second Edition; Revised by D. R. Heath-Brown. Clarendon Press Oxford, 1986.

[5] E. Carneiro, V. Chandee, M. B. Milinovich, Bounding $S(t)$ and $S 1(t)$ on the Riemann hypothesis, Math. Ann. Vol. 356, Issue 3 (2013), pp. 939-968.

[6] E. Carneiro, V. Chandee, M. B. Milinovich, An note on the zeros of zeta and $L$-functions, Math. Z., 281 (2015), 315-332.

[7] D. A. Goldston and S. Gonek, A note on $S(t)$ and the zeros of the Riemann zeta funcion, Bull. London Math. Soc. 39, (2007), 482486. 